\title{
A prática de atividade física na cidade universitária da Universidade Federal de Mato Grosso do Sul (UFMS)
}

\author{
The practice of physical activity in the campus \\ of Universidade Federal de M ato Grosso do Sul (UFM S)
}

Caroline Silva de Oliveira ${ }^{1}$

Claudia A parecida Stefane ${ }^{1}$

Leonardo Liziero ${ }^{1}$

Jarina Gomes Gabilan ${ }^{1}$

Antonio Conceição Paranhos Filho ${ }^{1}$

Fernanda Karolline de O liveira Guimarães ${ }^{1}$

${ }^{1}$ Departamento deEducação Física, Universidade Federal de M ato Grosso do Sul. Rua Elesbão M urtinho 8, Bairro Universitário. 79071-203 Campo Grande MS. carolsobike@hotmail.com
Abstract Theimplementation and strengthening of initiatives on physical activity and health are needed to guide and propose body practice in a conscious and autonomous way. Given the relevance of the investigation of places to the practice of physical activity in the development of public policies and practices of intervention, it is necessary knowledge about where and how this activity is developed, and the characteristics of the peopleinterested. Theobjective of thisstudy was characterize practitioners of physical activity in the Campus of Universidade Federal de $M$ ato Grosso do Sul (UFM S); identifying ways and reasons that led these people to seek for that space and the importance of vocational guidance for the practice. Data was collected by observation and interview with 75 people in N ovember 2006. The results indicated that most were men, adults, practiced mostly to walk without professional guidance. It is hoped that studies is encouraging the development of projects in recognition of where and how physical activity is developed and characteristics of who the search. This information can collaborate to interpretations based, interventions and initiatives in public policy, as they may drive development of programs to encourage physical activity, such as municipal Atlas of Sports, in operation. Key words Physical activity, H ealth, Life quality, Atlas of the sport
Resumo A implementação e o fortalecimento de iniciativas na atividade física e saúde são necessárias para orientar e propor práticas corporais de forma consciente e autônoma. Diante da relevância de levantamento de locais para prática de atividade física na elaboração de políticas públicas e práticas de intervenção, se faz necessário 0 conhecimento sobre onde e como esta atividade é desenvolvida, além das características de quem a busca. Este estudo teve por objetivos caracterizar os praticantes de atividade física na Cidade U niversitária da U niversidadeFederal deM ato Grosso do Sul (UFM S), identificar as modalidades e motivos que os levavam a procurar aquele espaç̧o ea importância de orientação profissional para esta prática. A coleta ocorreu por observação e entre vista com 75 indivíduos, em novembro de 2006. 0 s resultados indicaram que a maior parte eram homens, adultos, praticantes de caminhada enão tinham orientação profissional. Espera-se que estudos sejam realizados, pois estas informações podem colaborar para intervenções e iniciativas de políticas públicasfundamentadas, além de direcionar a elaboração de programas de incentivo à atividade física, como o A tlas do Esporte em âmbito municipal (em operacionalização).

Palavras-chave Atividade física, Saúde, Qualidade de vida, Atlas do esporte 
Introdução

A atividade física sempre existiu na história da humanidade e tem relação com as modificações ocorridas no processo de formação da sociedade. Estudos antropológicos e evidências históricas relatam a existência desta prática desde a cultura pré histórica, como um componente integral da expressão religiosa, social e cultural ${ }^{1}$.

Pela sua relação inversa com as doenças degenerativas, a atividadefísica éuma área relevantede investigação, pois indivíduos ativos tendem a apresentar menor mortalidadeemorbidade por doenças respiratórias, cardiovasculares, diabe tes, artrites e reumatismos². Dessa forma, várias instituições e organizações, como a American H eart Association ${ }^{3}$, a Organização Mundial de Saúde 4 e o Colégio Americano de M edicina Desportiva ${ }^{5}$, têm enfatizado a importância da adoção de atividade física regular para a melhoria dosníveis de saúdeindividual ecoletiva, especialmente para a prevenção e reabilitação de doenças cardiovasculares.

$A$ atividade física é definida como qualquer movimento corporal produzido pela musculatura esquelética, portanto voluntário, que resulte num gasto energético acima de níveis de repouso, que envolve, por exemplo, a realização de atividades domésticas e de lazer; um exemplo de atividade física é uma caminhada a passos rápidos por trinta minutos, realizada cinco vezes por semana ${ }^{6}$.

Este estudo direciona-se no eixo da atividade física pela qualidade de vida. Segundo $N$ ahas ${ }^{7}$, é difícil definir qualidade de vida de forma objetiva, mas numa visão geral, ela é a condição humana diante de um conjunto de parâmetros individuais esocioambientais, modificáveisou não, quecaracterizam as condições em que vive o ser humano ${ }^{7}$.

Ao que se refereà prática de atividades físicas, o governo brasileiro formulou a Política Nacional de Promoção da Saúde (PNS) ${ }^{8}$ visando modificar os altos índices de sedentarismo, ampliar e qualificar o acesso às práticas corporais no âmbito da atenção à saúde e produzir estratégias de melhoria da qualidade de vida da população?.

A PNS reforça a importância de se trabalhar pela autonomia e qualidade de vida dos cidadãos, possibilitando a construção de modos de viver favoráveis à saúde $\mathrm{e}^{10,11}$.

Existem estudos importantes enfocando indivíduos, grupos e comunidades ${ }^{2-5}$. Entretanto, nem todas as questões estão adequadamenterespondidas nesta área, em particular quando se refere à população brasileira.
Entre os pontos em debate está aquele relativo aos dados sobre o nível de atividade física do brasileiro, uma vez que pou cos são os estudos de âmbito nacional com este enfoque $e^{12-14}$.

A importância desses dados está na sua utilização como referência em políticas e programas de saúde pública na promoção da atividade física; portanto, precisam ser representativos eatualizados periodicamente?.

Pesquisadores voluntários mapearam as atividades físicas no Brasil em quase trezentas abordagens diferentes, desde esportes preferidos até laboratórios, passando por clubes e turismo de lazer esportivo. Baseado neste mapeamento, foi elaborado o Atlas do Esporte no Brasi ${ }^{15}$, que põe em relevo as atividades esportivas e seus suportes institucionais e comunitários como um dos principais meios para o desenvolvimento social eeconômico da sociedade brasileira na atualidade. Porém, verifica-sequenão existeno Brasil um sistema de informações esportivas ou sobre práticas de atividades físicas em geral, o que impede e dificulta intervenções adequadas ou políticas públicas coerentes ${ }^{16}$.

Para isso, é importante mapear aspectos que envolvem a atividade física como, por exemplo, o Atlas do Esporte no Brasi ${ }^{17}$. Democratizar e universalizar 0 direito à educação física exige 0 estabelecimento de metas, a coordenação de esforços públicos e privados, a elaboração de programas e eventos com vistas a maximizar o re torno social ${ }^{15}$.

A implementação e o fortal ecimento deiniciativas no campo da atividade física e saúde são necessárias para que trabalhadores dessas áreas tenham condições de orientar e propor práticas corporais ao cidadão de forma segura e eficaz.

Considerando a relevância de mapeamentos de locais para prática de atividade física na elaboração de políticas públicas e práticas de intervenção, se faz necessário o conhecimento sobre onde e como esta atividade é desenvolvida e as características da população que a busca.

Esta pesquisa está inserida no projeto " $\mathrm{Ma}$ peamento e descrição das condições dos espaços públicos onde é possível a prática de atividades físicas em Campo Grande - M S", que tem como objetivo mapear os espaços públicos disponíveis na área urbana de Campo Grande para a prática de atividade física e as condições destes espaços.

Diante desse contexto, este estudo tem por objetivos caracterizar os praticantes de atividade física na Cidade Universitária da Universidade Federal de M ato Grosso do Sul (UFM S); descrever as atividades realizadas nesse local; identifi- 
car os motivos que os levam a procurar esse espaço ea opinião sobrea importância de orientação profissional para realização das mesmas.

\section{M étodo}

Tipo de pesquisa

0 presente artigo caracteriza-se por uma pesquisa exploratória de caráter transversal. Exploratória, pois envolve levantamento bibliográfico, entrevistas com pessoas que têm experiências práticas com o problema pesquisado eanálise de exemplos que estimulem a compreensão. Dessa forma, este tipo de estudo visa proporcionar um maior conhecimento para o pesquisador acerca do assunto, a fim de que esse possa formular problemas mais precisos ou criar hipóteses que possam ser pesquisadas por estudos posteriores ${ }^{18}$. Transversal, visto que a coleta de dados ocorreu somente no mês de novembro de 2006.

\section{Local}

A Cidade Universitária da Universidade Fe deral de M ato Grosso do Sul (UFM S), localizada na cidade de Campo Grande, possui como estrutura esportiva um estádio de futebol, pista de atletismo, pista decaminhada, ginásio poliesportivo, quadras poliesportivas, sala de musculação e complexo aquático.

\section{Participantes do estudo}

A população-alvo desse estudo foi composta pelos praticantes de atividade física (AF) na $\mathrm{Ci}$ dade Universitária da UFMS.

A amostra foi composta por 75 indivíduos que realizavam $\mathrm{AF}$ entre seis e oito da manhã; cinco e seis e meia da tarde, pois foram os horários em que se constatou maior presença do público-alvo.

\section{Instrumento}

As informaç̃̃es foram obtidas por meio de roteiro de observação e de entrevista. 0 roteiro de observação, composto de três itens, tinha por objetivo identificar as características de praticantes de $A F$ na população do estudo quanto à vestimenta para prática de AF, considerando roupas que permitissem a execução do movimento de modo confortável e adequado para aquela prática (roupas leves como bermuda, malhas, camisetas); o uso de calçados adequados, desconsiderando os que estavam de sapatos com saltos, sandálias ou descalços e proteção contra o sol, em que foi considerado o uso de boné ou roupa com manga comprida.

Importante salientar que a proteção contra o sol foi um aspecto observado tendo em vista a região ter alta incidência de raios ultra-violeta (IVU) nesse período do ano, com registros de IUV acima de $13^{19}$, e este ser um fator de risco para o desenvolvimento do câncer de pele, o tipo mais incidente na maioria das regiões do Brasi ${ }^{20}$.

0 roteiro de entrevista foi composto por dezesseis questões que abordaram as variáveis sociodemográficas - sexo, idade, profissão, nível de escolaridade, renda mensal ebairro em que residia -, além de variáveis da prática AF: existência de locais para prática AF no bairro em que residia, atividade praticada dentro e fora da UFMS, frequência da $A F$, motivo da busca pela $A F$ e re conhecimento da importância da orientação profissional para a prescrição de AF.

\section{Procedimentos}

Para realização do estudo piloto, os instrumentos foram aplicados em indivíduos com características idênticas aos que configuram na amostra deste estudo. A partir dos dados do teste piloto, observaram-se questões que não corresponderiam às expectativas do objetivo deste estudo, sendo então descartadas e/ou alteradas.

A entrevista foi realizada através de abordagem direta aos indivíduos que se caracterizavam como praticantes de atividades físicas. Após a abordagem inicial, o entrevistador explanava brevemente o objetivo do estudo ao entrevistado, que posteriormente autorizava a utilização de suas informações apenas para fim de consolidação desta pesquisa e respondia às questões solicitadas voluntariamente.

Após a autorização do participante, a observação era realizada eositens registrados em planilha.

\section{Análise estatística}

Os dados obtidos, tanto do teste piloto como da amostra oficial, foram submetidos à análise estatística descritiva (frequência, percentual, mé dia e desvio padrão) e para isto foi utilizado o software SPSS versão 10.0. 


\section{Resultados ediscussão}

Os participantes do estudo foram 75 indivíduos. Destes, 46 eram homens e 29 eram mulheres. $\mathrm{Na}$ Tabela 1, estão expostos os dados de observação.

O bserva-se que a maioria vestia-se e calçavase adequadamente para a AF. Foram encontradas pessoas que vestiam (5) e calçavam (12) vestimentas fora dos padrões recomendados para a prática de AF.

U ma minoria estava protegida contra os raios solares. 0 baixo uso de proteção solar pode ter ocorrido em função da prática de AF estar ocorrendo em um horário em que os raios solares são menos intensos. No entanto, no período de coleta, o estado estava seguindo o horário de verão, o que representava a realização da AF no sol das 16 horas.

Além dos dados de observação, procurou-se estimar o perfil do público do estudo. Dos 75 participantes, 46 eram homens e 29, mulheres, apontando uma diferença numérica relativamente pequena entre participantes do sexo masculino e feminino. Porém, a predominância masculina na prática de AF tem sido observada em outros estudos populacionais realizados em países desenvolvidos ${ }^{15}$ e no Brasil ${ }^{16,17}$.

Em geral, existe uma tendência de as mulheres praticarem menos AF quando comparadas aos homen $\mathrm{s}^{21}$, sendo este comportamento observado desdea infância eadolescência ${ }^{22}$. No Brasil, resultados semelhantes também têm sido descritos $^{23}$; no entanto, ainda são pouco conhecidos os fatores associados a esta menor participação das mulheres em atividades físicas.

Werneck et al..$^{24}$, em seu estudo, encontraram resultados semelhantes, visto que apenas um terço das mulheres praticava algum tipo de AF, enquanto quase $40 \%$ dos homens realizavam al guma AF. No estudo de Gomes et al. ${ }^{13}, 36,8 \%$ dos homens participavam de atividadefísica, enquanto entre as mulheres o percentual era de apenas 19,3\%. Em pesquisa realizada com funcionários deuma empresa estatal, Oliveira ${ }^{23}$ observou, tam-

Tabela 1. Dados resultantes da observação dos frequentadores.

\begin{tabular}{lc}
\hline \multicolumn{1}{c}{ Observação } & Porcentagem \\
\hline Roupa adequada & $93 \%$ \\
Calçado adequado & $84 \%$ \\
Proteção ao sol & $27 \%$ \\
\hline
\end{tabular}

bém, que as mulheres realizavam menos atividade física do que os homens.

H ouveelevada variação na idade dosfrequentadores, pois a mínima encontrada foi decatorze anose a máxima de 68 anos, A média deidadefoi de 38 anos e $48 \%$ se encontravam na faixa etária entre dezoito e 36 anos.

Pode-se constatar que, hoje em dia, a prática regular de atividade física é muitas vezes encarada como "mecanismo de prevenção" 25. Para que essa prevenção ocorra, é importante agir na fase em que se inicia a maior parte dos decréscimos fisiológicos - a vida adulta intermediária (dequarenta a sessenta anos) ${ }^{26}$.

Conforme constatou este estudo, a maioria dos indivíduos encontrava-sena faixa etária abaixo da fase adulta intermediária (menos de quarenta anos). Tamayo et al. ${ }^{27}$ relataram em seu estudo que para o adulto aderir à atividade física é mais complicado, pois eletem que abrir espaço na sua agenda para praticá-la, e se esta exigir uma infraestrutura determinada, procurar uma academia, um clube, um parque ou outro local apropriado, as barreiras para a prática começam a se multiplicar, fator este que pode explicar a menor presença de indivíduos desta faixa etária na Cidade Universitária.

As profissões citadas entre os 75 indivíduos foram estudante (16), funcionário público (14), autônomo (12), do lar (10), comerciante (4), aposentado (3), auxiliar administrativo (2), motorista (2), professor (2), digitador (2), economista, auxiliar de estoque, advogado, engenheiro agrimensor, costureira, cabeleireiro, auxiliar de enfermagem, encarregado, manicure, enfermeiro padrão, pintor, biólogo, gerente, contador, jardineiro. Como se pode observar, a escolha do local da prática de AF está relacionada ao local de atuação profissional, visto que 22 indivíduos trabalhavam ou estudavam na própria Cidade Universitária.

O nível de escolaridade dos frequentadores foi outro dado levantado neste estudo, porém os resultados apontaram para uma defasagem nas respostas, visto que indivíduos (52) não responderam à essa questão; ensino superior (13); ensino fundamental (6) e ensino médio (4).

Em relação à renda, a maioria ( 31 dos 75 entrevistados) se enquadrava entre dois e cinco saláriosmínimos; dezenove declararam rendamensal acima de cinco salários mínimos, dezoito afirmaram ter renda de até dois salários mínimos e sete não souberam responder.

A renda per capita foi outra variável associada àAF. Na população de estudo, indivíduos com 
maior renda per capita configuraram como os que mais praticavam atividades físicas. Resultado semelhante foi observado em uma amostra representativa de indivíduos da Alemanha ${ }^{28}$, na qual homens e mulheres com maior nível socioeconômico estiveram, respectivamente, quatro e três vezes mais engajados em atividades de lazer, independente de outros fatores relacionados ao estilo de vida. Ford et al..$^{29}$ encontraram, também, associação entrenível socioeconômico eAF, sendo homens e mulheres com menor nível socioeconômico menos ativos fisicamente.

Quanto à localização da residência dos praticantes, 27 bairros da cidade foram citados. A maioria ( 65 indivíduos - 80\%) residia em dezessete bairros localizados próximos à UFMS, de nominada Região Urbana do Bandeira, do município de Campo Grande (MS).

Os resultados indicaram que $59 \%$ dos indivíduos não praticavam AF em seu bairro, pois residiam próximosàUFMS, inexistiam locais deprática no bairro, os locais estavam em más condições de uso e não havia infraestrutura adequada.

Frente a isso, a questão seguinte queria identificar os motivos que os levaram a praticar AF na UFM S. Os motivos citados foram lazer (28), perto de casa (19), recomendação médica (11), falta opção no bairro (11), gratuidade (7), amigos (3), saúde (1), ambiente agradável (1) e perto do trabalho (1).

M ais da metade dos indivíduos analisados moravam em bairros ao redor da U FM S e relataram que na região onde residiam os locais para prática de AF eram geralmente inacessíveis à população, pois eram privados e estavam em más condições de uso. Interessante observar que, apesar de oito indivíduos estudarem ou trabal harem na UFM S, apenas um justificou isto como motivo; além disto, somenteum declarou queo ambiente da UFM S era agradável, por ser arborizado e calmo.

Diantedesses dados, tem-seum indicativo de escassez de locais públicos de fácil acesso ou que ofereçam uma infraestrutura capaz de proporcionar a busca saudável e correta pela qualidade de vida, principalmente em regiões próximas à universidade. Os espaços para a prática de AF são muitas vezes destinados à elite da população e estão concentrados geograficamente, além do que quase a totalidade das instalações existentes apresenta problemas de manutenção ${ }^{30}$. A pesar das inúmeras atividades promovidas pela Pre feitura de Campo Grande nos vários parques públicos existentes, estes resultados explicitam faIhas nas políticas e programas de incentivo à prá- tica de AF, ou seja, as atividades podem estar sendo oferecidas em locais inadequados ao público que deseja e procura praticar AF.

O Gráfico 1 mostra as modalidades de AF mais citadas pelos frequentadores da Cidade Universitária.

Entre as diversas modalidades de AF que poderiam ser praticadas na Cidade Universitária, apenas nove (9) foram apontadas: corrida, caminhada, natação, hidroginástica, futsal, futebol, voleibol, basquetebol, patinação exadrez. Asmais frequentes foram caminhada (38), futsal (11), basquetebol (10) enatação (9). Futebol, voleibol, patinação exadrez foram citadas somente ma vez.

A caminhada foi a modalidade de AF mais frequente entre os entrevistados. Dentre os motivos para explicar essa preferência, tem-seo fato de ser uma modalidade individual, exigir pouco em termos de equipamentos, poder ser feita em qualquer lugar e ser considerada uma atividade simples, o que não quer dizer que as pessoas a fazem corretamente. É uma modalidade recomendada quase que unanimemente pelos profissionais da saúde. Sabe se que éum dos exercícios aeróbios mais adequados para adultos, especialmente idosos e portadores de doenças cardíacas, porém apesar da população conhecer os seus benefícios, a caminhada pode ser ao mesmo tempo prejudicial, uma vez que sua prática ére alizada sem orientação e de manei ra incorreta.

A Cidade Universitária possui uma estrutura física favorável à realização deAF eédefácil aces-

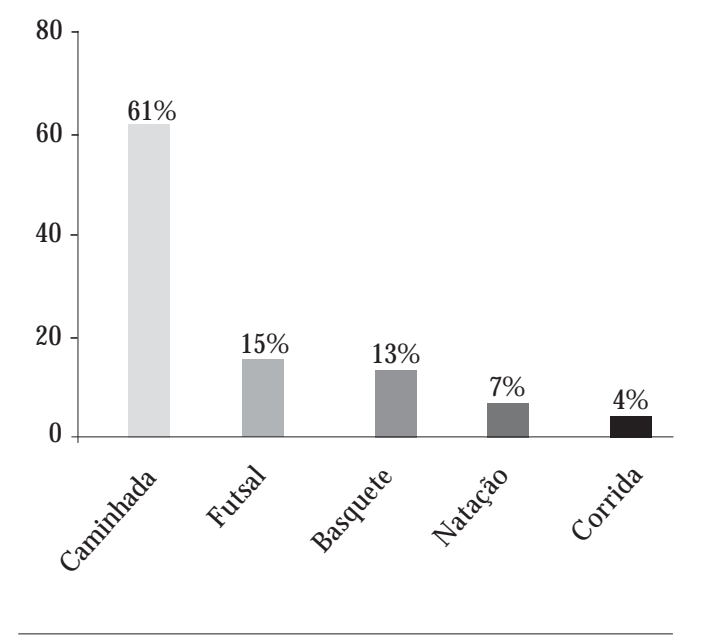

Gráfico 1. M odalidades de AF mais citadas pelos entrevistados. 
so à comunidade, não só acadêmica, como externa. De acordo com a observação e análise das respostas, nota-se que essa estrutura está sendo subutilizada, uma vez que no período da coleta foi observada a baixa utilização, principalmente dos locais destinados à prática de esportes coletivos ou que necessitem de materiais como bolas e redes. A universidade não disponibiliza materiais esportivos para os frequentadores; entretanto, por meio de projetos de extensão como futsal, voleibol, natação e hidroginástica, a UFM S ofereceà comunidadeexterna a oportunidadede praticar $A F$, inclusive aos fins de semana.

N os fins de semana, a prática de esportes coletivos eas atividades de lazer em família tiveram uma aderência maior em relação ao restante da semana, ou seja, os indivíduos levavam seus próprios materiais para a prática. Durante a coleta, observou-se um grupo de amigos jogando voleibol no estacionamento. Eles utilizavam duas árvores como postes para praticar aquele esporte. Ao questioná-los sobre o porquê de jogar naquelelocal, eles disseram quefrequentavam o local todo fim de semana e o escolheram por ser mais calmo e não terem que disputar a quadra com outras pessoas.

No Gráfico 2, estão expostos dados a respeito do recebimento ou não de orientação profissional para a prática de AF.

Conforme se observa no Gráfico 2, a prática da AF era realizada sem orientação profissional por mais da metade dos indivíduos. Dos 25 que receberam orientação, catorze responderam que esta veio por parte de médicos, dez, por profissi-

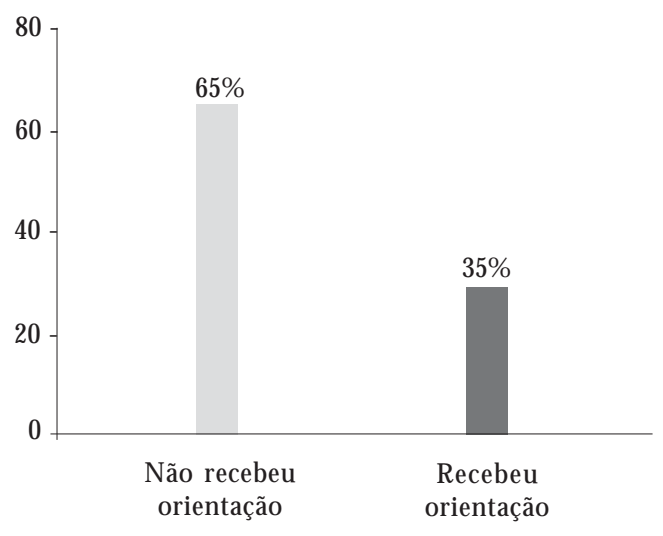

Gráfico 2. Recebimento ou não de orientação sobre como realizar AF. onais de educação física e um, por militar. Esses dados revelam que a busca pela AF ocorre sem orientação de profissionais capacitados a prescreverem atividades, assim como muitos praticam AF sem um programa adequado, o quepode ocasionar tanto lesões quanto a não obtenção dos objetivos desejados.

0 Gráfico 3 apresenta os resultados relacionados à importância do profissional de educação física na prescrição e orientação da AF a ser praticada.

A presença do profissional de educação física na organização e condução da AF foi vista como fundamental para a maioria dos entrevistados (69). Pode-se inferir que a mai oria dos indivíduos tinha consciência da importância deste profissional para a prática regular de exercícios físicos; entretanto, a maioria deles não buscou ajuda na sistematização de sua AF e a realiza de maneira autônoma, o que pode acarretar efeitos opostos aos intencionados.

Ao perguntar aos indivíduos como a presença do profissional de educação física poderia ajudá-lo, eles indicaram situações como orientação à real ização do exercício (50), prescrição do plano de atividade (30), motivação (11) e avaliação (3).

A pesar dessa evidência em relação à visão dos praticantes de AF sobre a importância da pre sença do profissional de educação física, outra questão apresentou um contraponto: dos 69 indivíduos que consideravam importante a pre sença do profissional de educação física, quarenta não estavam dispostos a pagar por esse serviço e 29 pagariam. Entre os 29 que pagariam pe-

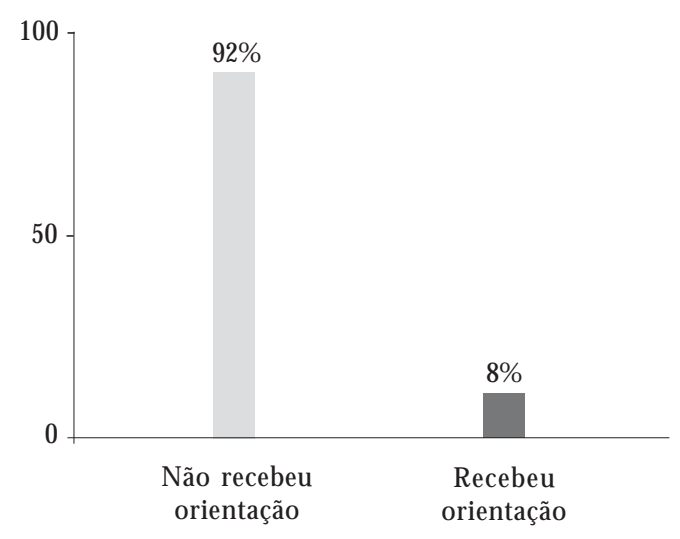

Gráfico 3. Importância do profissional de educação física na prescrição da AF. 
los serviços, dezoito disseram que desembolsariam até $R \$ 20,00$ reais mensais.

Isto denota que a maioria dos participantes tem conhecimento sobre as atribuições do profissional de educação física; no entanto, não o reconhece como profissional que se mantém no mercado de trabalho, visto a não disposição de pagamento pela orientação profissional do educador físico por mais da metade dos indivíduos.

Diante disto, cabe à classe profissional da educação física prestar esclarecimentos à sociedade sobre suas atribuições, valorizando assim o seu papel na sociedade.

\section{Consideraçõesfinais}

Os dados resultantes do roteiro de entrevista permitem ter o conhecimento de uma parcela da população da cidade de Campo Grande que pratica AF. Apesar de ser um público pequeno se comparado à população brasileira, a partir desses dados, pode-se desenvolver uma referência de informações que possibilitem intervenções adequadas e coerentes quanto ao incentivo à prática de AF.

Salienta-se a importância dese trabalhar pela autonomia e qualidade de vida dos cidadãos, ampliando as suas possibilidades de construir modos de viver favoráveis à saúde e que reduzam sua vulnerabilidade ao adoecimento ${ }^{11,31}$.

Daí a importância de se conhecer quem frequenta um local onde é possível praticar AF, 0 que eles fazem, o que os levam a se deslocarem até lá, até que ponto o profissional de educação física é visto como importante para os cidadãos, para que este possa intervir de maneira a proporcionar à população o direito à busca pela qualidade de vida através da AF.

A partir do Atlas do Esporte, verificou-seque não existe no Brasil um sistema de informações esportivas ou de práticas de atividades físicas em geral, impedindo assim que haja intervenções adequadas ou políticas públicas coerentes. Neste contexto, o objetivo de se instituir a coleta de dados sobre AF de modo sistemático no Brasil está sendo reforçado.

Projetos de pesquisa como “Análiseespacial dos dados da Pesquisa do Esporte 32 por municípios do Estado de M ato Grosso do Sul"; "Levantamento e mapeamento dos espaços para atividade física no Estado de M ato Grosso do Sul" e "M apeamento e levantamento dos espaços para atividade física na Região Imbirussu em Campo Grande/M S" por meio de geotecnologias, alimentam o banco de dados sobre esporte, atividade física e educação física no Estado de M ato Grosso do Sul.

Além da identificação dos locais para a prática de AF em Campo Grande, caracterizar os praticantes que buscam os espaços públicos da cidade para a prática de AF se torna fundamental para a elaboração do Atlas MS.

Diante deste contexto, pode-secaracterizar os praticantes de AF na UFM S como homens, adultos, praticantes decaminhada, tinham por objetivo a busca pelo lazer, renda mensal entre dois ecinco salários mínimos, que não receberam orientação profissional para a prática de AF e tampouco estavam dispostos a pagar por este serviço.

Considerando os resultadose o fato daU FM S possuir o curso de educação física eter instituído - Programa de Atividades Físicas (PAF), que por meio de atividades de extensão oferece modalidades esportivas (futsal, basquetebol, voleibol, lutas, natação), danças, ginástica, além do xadrez e hidroginástica para a comunidade acadêmica e sociedade em geral, questiona-se o não oferecimento de orientação ao público caracterizado neste estudo.

Instituições de ensino superior, órgãos públicos federais, estaduais e municipais e outras organizações têm em suas mãos instrumentos fundamentais para atuarem em seus papéis de mediadores entre o conhecimento e a sociedade, enfim, podem incentivar a prática de AF.

Espera-se que este estudo contribua para a elaboração de projetos relativos à promoção da $A F$, visto que estas informações podem colaborar para interpretações fundamentadas de lideranças e gestores governamentais em suas intervenções e iniciativas de políticas públicas sobre saúde e educação física, assim como direcionar a elaboração de programas de incentivo à $A F$, tanto em locais privados como públicos.

\section{Colaboradores}

CS Oliveira trabalhou na concepção, redação e aprovação do artigo; CA Stefane trabalhou na concepção e aprovação do artigo; AC Paranhos Filho trabalhou no delineamento eaprovação do artigo; FKO Guimarães, JG Gabilan e L Liziero trabalharam na redação do artigo; MS Procópio, CL Rocha eLM Rocha trabalharam na coleta de dados. 
1. US Department of Health and Human Services. Surgeon General's report on physical activity and health. From the Centers for Disease Control and Prevention. JAM A 1996; 276(7):522.

2. Centers for Disease Control and Prevention. $\mathrm{Na}$ tional Center for Chronic Disease Prevention and Health Promotion. Physical Activity and Health. A Report of the Surgeon General. Atlanta: CDC; 1996.

3. Fletcher GF, Blair SN, Blumenthal J, Caspersen C, Chaitman B, Epstein S, Falls H, Sivarajan ES, Froelicher VF, Pina IL. Statement on exercise. Benefits and recommendations for physical activity programs for all Americans. Circulation 1992; 86:341-344.

4. Bijnen F, Caspersen C, Mosterd W. Physical activity as risk factor for coronary heart disease: A WHO and International Society and Federation of Cardiology position statement. Bull World Health Organ 1994:72(1) 1-4.

5. Pate R, Pratt M, Blair SN, Haskell W, Macera CA, Bouchard C, Buchner D, Ettinger W, Heat G, King AC, Kriska A, Leon AS, M arcus BH, M orris J, Paffenbarger RS, Patrick K, Pollock ML, Rippe JM, Sallis J, Wilmore JH. Physical activity and public health: a recommendation from the Centers for Disease Control and Prevention and the American College of Sports M edicine. JAM A 1995; 273:402-407.

6. Caspersen CJ, Powell KE, Christensen GM. Physical activity, exercise, and physical fitness: definitions and distinctions for health-related research. Public Health Reports 1985; 100:126-131.

7. Nahas MV. Atividade física, saúde e qualidade de vida. 4a ed. Londrina: M idiograf; 2006.

8. Brasil. M inistério da Saúde. Política Nacional de Promoção da Saúde. Brasília: M inistério da Saúde; 2005.

9. Brasil. M inistério da Saúde. Minuta de Portaria que cria o N úcleo de Atenção Integral na Saúde da Família - NAISF. [site da Internet] 2005 [acessado 2007 abr 07]. Disponível em: http//portal.saúde.gov.br

10. Organização Mundial da Saúde. Estratégia Global em Dieta, Atividade Física e Saúde. 57ạ Assembléia M undial de Saúde. [site da Internet] 2004 [acessado 2007 abr 05]. Disponível em: http//ibesa.pt

11. World Health Organization. The world health report. [site da Internet]. 2000 [acessado 2007 abr 20]. Disponível em: http://www.who.int/whr/en/2000

12. M onteiro CA, Conde $W L, M$ atsudo $S M, M$ atsudo VR, Bonsenor IM, Lotufo PA. A descriptive epidemiology of leisure-time physical activity in Brazil, 1996-1997. Rev. Panam. Salud Públ. / Pan Am. J. Public Health 2003; 14:246-254.

13. Gomes VB, Siqueira KS, Sichieri R. Atividade física em uma amostra probabilística da população do município do Rio de Janeiro. Cad Saude Publica 2001; 17:969-976.

14. Santos AM M M, Gimenez LC, Rebouças CE, Schimitt SL, Rennó T. Esportes no Brasil: situação atual e propostas para desenvolvimento. [site da Internet]. 1997 [acessado 2007 abr 10]. Disponível em: http://www.bndes.gov.br/conhecimento/bnset/ esporte.pdf

15. Dacosta L, organizador. Atlas do Esporte no Brasil: atlas do esporte, educação física e atividades físicas de saúde e lazer no Brasil. Rio de Janeiro: Shape; 2005.
16. Costa DS, Machado LC, Sales V, Cruz R. Cref 11Conselho Regional de Educação Física do $M$ ato Grosso e M ato Grosso do Sul. In: Dacosta, L, organizador. Atlas do Esporte no Brasil: atlas do esporte, educação física e atividades físicas de saúde e lazer no Brasil. Rio de Janeiro: Shape; 2005. p. 54-157.

17. Instituto Brasileiro de Geografia e Estatística. [site da Internet]. 2007 [acessado 2007 jul 28]. Disponível em: http://www.ibge.gov.br/home/estatistica/indicadores/ trabalhoerendimento/pme/pmemet2.htm

18. Gil AC. M étodos e técnicas de pesquisa social. São Paulo: Atlas; 1999. p. 51.

19. Mato Grosso do Sul. Climatologia. [site da Internet]. [acessado 2007 out 10]. Disponível em: http:/ /www.ms.gov.br/noticias/clima

20. M ato Grosso do Sul. Índices. [site da Internet] [acessado 2007 nov 15]. Disponível em: http://www.ms. gov.br/noticias/indice

21. Manios Y, Kafatos A, Codrington C. Gender differences in physical activity and physical fitness in young children in Crete. J Sports M ed Phys Fitness 1999; 39:24-30.

22. Garcia AW, Pender NJ, Antonakos CL, Ronis DL. Changes in physical activity beliefs and behaviors of boys and girls across the transition to junior high school. J Adol Health 1998; 22:394-402.

23. Oliveira CCM . Atividade física de lazer e sua associação com variáveis demográficas e outros hábitos relacionados à saúde em funcionários de banco estatal [dissertação] Rio de Janeiro (RJ): Escola Nacional de Saúde Pública, Fundação Oswaldo Cruz; 2000.

24. Werneck GL. Associação entre fatores sócio-demográficos e prática de atividade física de lazer no Estudo Pró-Saúde. Cad Saude Publica 2003;19(4):123.

25. Moreira CA. Atividade física na maturidade. Rio de Janeiro: Shape; 2001. p. 42-46.

26. Santos SC, Knijnik, JD. M otivos de adesão à prática de atividade física na vida adulta intermediária I. Rev M ackenzie Educ Fis Esp. 2006; 5(1):23-34.

27. Tamayo A, Campos APM, Matos DR, Mendes GR, Santos JB, Carvalho NT. A influência da atividade física regular no auto-conceito. Rev Est Psicologia 2001; 6(2):157-165.

28. Mensink GBM, Loose N, Oomen CM. Physical activity and its association with other lifestyle factors. Eur J Epidemiol 1997; 13:771-778.

29. Ford ES, Merritt RK, Heath GW, Powell KE, Wasburn RA, Kriska A, Haile G. Physical activity behaviors in lower and higher socioeconomic status populations. Am J Epidemiol 1991; 133:1246-1256.

30. Caspersen CJ, Pereira MA, Curran KM. Changes in physical activity patterns in the United States, by sex and cross-sectional age. M ed Sci Sports Exerc 2000; 32(9):1601-1609.

31. Puska P. Community change and the role of public health. Geneva: WH O; 2003.

32. Instituto Brasileiro de Geografia e Estatística. Perfil dos municípios brasileiros Esporte 2003. [site da Internet] [acessado 2007 abr 06]. Disponível em: http:/ /www.ibge.br/perfil/esporte2003

Artigo apresentado em 31/01/2008

Aprovado em 28/10/2008

Versão final apresentada em 29/11/2008 